\title{
Change of Eye Position in Patients with Orthophoria and Horizontal Strabismus under General Anesthesia
}

\author{
Hee Chan $\mathrm{Ku}, \mathrm{MD}^{1}$, Se-Youp Lee, $\mathrm{MD}^{2}$, Young Chun Lee, $\mathrm{MD}^{1}$ \\ Department of Ophthalmology, Uijongbu St. Mary's Hospital, \\ College of Medicine, The Catholic University of Korea', Seoul, Korea \\ Department of Ophthalmology, Dongsan Medical Center \\ College of Medicine, Keimyung University of Korea², Deagu, Korea
}

\begin{abstract}
We studied the relationship between eye position in the awakened state and in the surgical plane of anesthesia in orthophoric and horizontal strabismus patients. We classified 105 orthophoric and horizontal strabismus patients into 5 groups, measured the eye position at the primary position by photographic measurement of the corneal reflex positions and undertook a quantitative study of eye position. Under general anesthesia, the mean divergence was 39.7 78 PD for the esotropia group, 36.6 \pm 11.7 PD for exophoria, 27.4 \pm 8.1 PD for orthophoria, and 11.1 \pm 10.2 PD for exotropia I $(\leq 30$ PD). Therefore, the esotropia group had the largest amount of divergence among the groups, but the eye position of the exotropia II ( $>30$ PD) group was rather convergent at 11.0 \pm 6.5 PD. According to the eye position of the fixating and nonfixating eyes in the esotropia group, both eyes converged with an angle deviation of $14.4 \pm 4.8$ PD divergent and 14.1 \pm 4.8 PD divergent, respectively ( $\mathrm{P}=.71$ ). In the exotropia groups (I, II), the fixating eye diverged but the nonfixating eye rather converged. Therefore, the angle deviation was 19.0 \pm 2.1 PD divergent for the fixating eye and 18.2 \pm 6.4 PD divergent for the nonfixating eye $(\mathrm{P}=\mathbf{6 8})$. In conclusion, under general anesthesia, eye positions in the awakened state and in the surgical plane of anesthesia were convergent or divergent, and showed a tendency to converge into the position of 25-35 PD divergent. Therefore, we could not distinguish fixating eye from nonfixating eye under general anesthesia.
\end{abstract}

Key words: Converge, Diverge, Photographic measurement

Generally, eye position after general anesthesia in orthophoria and strabismus has been reported to be divergent. $^{1-8}$ In 1890, Grut $^{1}$ reported that eye position during sleep, death, blindness, and after anesthesia is divergent, and in 1958 Moller $^{2}$ measured the change of eye position after ether anesthesia. Subsequently, numerous studies have

Reprint requests to Young Chun Lee, MD. Department of Ophthalmology, Uijongbu St. Mary's Hospital, College of Medicine, The Catholic University of Korea, \#65-1 Kumoh-dong, Uijongbu-si, Gyeonggi-do 480-130, Korea. Tel: 82-31-820-3110, Fax: 82-31-8473418, E-mail: leeyc@cmc.cuk.ac.kr

This study was presented at the $89^{\text {th }}$ Annual Meeting of the Korean Ophthalmological Society, April, 2003 and as a poster at the ARVO Annual meeting, 2004, Florida, USA.

A part of the printing expenses of this study was supported by the Catholic Medical Center, Seoul, Korea. reported the eye position after general anesthesia in strabismus and orthophoria patients, and even the correlation of eye position after general anesthesia has been reported. In Korea, it has been reported by Lee et $\mathrm{al}^{3}$, Kim et $\mathrm{al}^{4}$, An et $\mathrm{al}^{5}$, and Lim et $\mathrm{al}^{6}$ in strabismus patients, and by Lee and $\mathrm{Kim}^{7}$ on orthophoria. In these studies, eye position was measured during the awakened state by the alternative prism cover test, and after general anesthesia the squint angle was measured by Hirschberg or Krimsky prism test. Therefore, the shortcoming of these studies is the lack of objectivity in the comparison of eye position between before and after anesthesia. However, in 1987 Brodie $^{27}$ applied Hirschberg to enumerate the evaluation of eye position by taking the pictures of the front view and quantitatively measuring the corneal reflex position. This method is known to be more 
objective for evaluating eye position and has been reported in Korea by Lee and Lee. ${ }^{26}$

In our study, pictures of horizontal strabismus patients and orthophoria individuals in the awakened state and under general anesthesia were taken by digital camera, the corneal reflex position was assessed by measuring the distance based on the nasal limbus, and the change of the monocular position was also measured by distinguishing between the fixating and nonfixating eyes. In addition, it was examined whether the eye position during the awakened state correlated to that under general anesthesia. Further investigation included the change of the position of both eyes in orthophoria, and the change of the position of both nonfixating and fixating eyes in strabismus patients.

\section{MATERIALS AND METHODS}

The study was performed from August 2002 to March 2003 on 30 patients with orthophoric eye position, 30 exophoria patients who underwent surgery for horizontal strabismus, 30 exotropia patients, and 15 esotropia patients (total 105 patients). Patients with a history of extraocular muscle surgery, neurological diseases, and accompanied vertical strabismus were excluded from the study population. The age distribution was from 4 years to 38 years with a mean age of 12.5 years.

The squint angel was measured one day prior to surgery by asking the patients to stare at the focus of the camera at $40 \mathrm{~cm}$ distance in front, placing a ruler on the forehead, and taking pictures. At the time of photography, the corneal

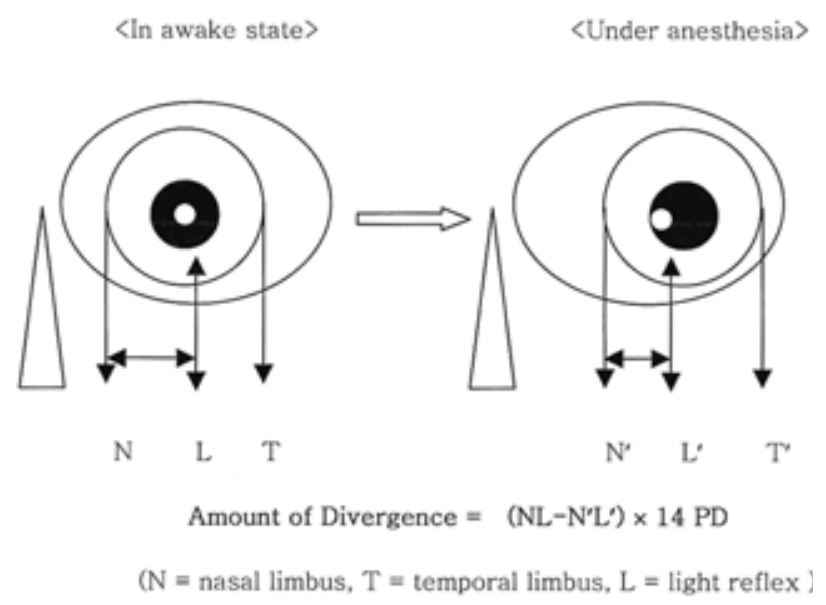

Fig. 1. Schematic illustration of eye position in both the awakened state and the surgical plane of anesthesia. reflex was almost on the center of the cornea of orthophoria and exophoria (intermittent exotropia), that of the fixating eye was on the center of the cornea and that of the nonfixating eye was skewed from the center of the cornea to the nasal limbus in exotropia patients, whereas in esotropia patients that of the fixating eye was in the center of the cornea and that of the nonfixating eye was skewed from the center of the cornea to the lateral limbus.

Anesthesia was induced after the inhalation of oxygen by administering $5 \mathrm{mg} / \mathrm{Kg}$ pentobarbital and $1 \mathrm{mg} / \mathrm{Kg}$ succinylcholine, after which endotracheal intubation was performed. Anesthesia was subsequently maintained by carbon dioxide and oxygen, and 20-30 minutes after anesthesia the muscle relaxant vecuronium $(0.1 \mathrm{mg} / \mathrm{Kg})$ was administered, and eye position was measured. The eye position under general anesthesia was measured by placing a ruler on the spot 40 $\mathrm{cm}$ vertically above the glabella of the patient, and taking pictures by digital camera.

The calculation of the squint angle was measured by defining the middle of the nasal limbus and lateral limbus as the center of the cornea, the reflex spot on the cornea was determined. Subsequently, in the orthophoria group, the distance from the center of the cornea and the corneal reflex was measured, whereas in the strabismus eyes, the distance from the nasal limbus to the corneal reflex was subtracted from half of the corneal diameter, and the difference before and after anesthesia was calculated by the difference of the distance from the nasal limbus to the corneal reflex spot. The results in millimeters were multiplied by 14 according to the conventional conversion rate of 14 Prism diopter/ $\mathrm{mm},{ }^{14}$ and taken as the squint angle. In all calculations, the right and left eyes were calculated separately and subsequently added (Fig. 1).

Statistical analysis was performed using SPSS 11.5, the mean value within each group was compared by t-test and Mann-Whitney test, the mean value among each group was compared by Kruskal-Wallis one-way ANOVA, and $p$ value less than 0.05 was considered statistically significant.

\section{RESULTS}

In the orthophoria group, the eye position before anesthesia was $3.9 \pm 3.5 \mathrm{PD}$ and after anesthesia was $31.4 \pm$ 7.6 PD, giving a divergence of $27.4 \pm 8.1 \mathrm{PD}$. In the exotropia group, before anesthesia was 5.6 \pm 3.5 PD and 
after anesthesia was $34.0 \pm 4.6 \mathrm{PD}$, giving a divergence of 36.6 \pm 11.7 PD. Among the 30 orthophoria patients, 29 cases showed positive angle kappa, and 1 (3.33\%) showed negative angle kappa.

In the exotropia group, dependent on the degree of strabismus during the awakened state, a tendency toward changing eye position to different directions after anesthesia was detected. In exotropia under 30 PD (exotropia I group), it was primarily divergent after anesthesia, while in exotropia over 30 PD (exotropia II group), a convergent tendency was detected. In the exotropia I group, before anesthesia was $24.9 \pm 4.7 \mathrm{PD}$ and after anesthesia was 36.0 $\pm 9.1 \mathrm{PD}$, giving a divergence of $11.1 \pm 0.2$ PD. In the exotropia II group, before anesthesia was 47.1 \pm 6.5 PD and after anesthesia was $36.6 \pm 5.2 \mathrm{PD}$, giving a convergence of $11.0 \pm 6.5$ PD. In addition, in the esotropia group, the convergence was $39.7 \pm 8.0 \mathrm{PD}$, from before anesthesia of 17.9 \pm 6.4 PD to after anesthesia of $21.8 \pm 7.5 \mathrm{PD}$, that was the largest among the 5 groups. The exotropia II group had a convergence of $11 \pm 6.5$ PD. There was no statistically significant difference among the 4 groups after the esotropia group was excluded $(\mathrm{P}=0.137)$. However, there was a significant difference among all 5 groups $(\mathrm{P}=0.006)$, although since nonparametric tests were performed, post-hoc tests could not be performed. Finally, in all orthophoria and horizontal strabismus patients, the eye position after general anesthesia showed a divergent tendency of 25-35 PD (Table 1 and Figs. 2 and 3).

In the orthophoria group, before anesthesia the right eye was $0.2 \pm 0.1 \mathrm{PD}$ and the left eye was $0.3 \pm 0.1 \mathrm{PD}$, and after anesthesia it was $13.6 \pm 6.6 \mathrm{PD}$ and $13.6 \pm 5.0 \mathrm{PD}$, giving divergences of $13.4 \pm 6.5 \mathrm{PD}$ and $13.3 \pm 4.9 \mathrm{PD}$, respectively. The difference of divergence between the two eyes was not significant $(\mathrm{P}=0.45)$ (Table 2).

In the exotropia group, measuring the fixating and nonfixating eyes separately, before anesthesia the fixating eye was $1.7 \pm 3.6 \mathrm{PD}$ and the nonfixating eye was $34.3 \pm$ 12.9 PD, with the difference between the two eyes being significant $(\mathrm{P}<0.01)$. After anesthesia, the fixating eye showed divergence of $17.3 \pm 5.7 \mathrm{PD}$ and the nonfixating eye showed convergence of $16.1 \pm 6.5 \mathrm{PD}$, and the divergent position of the fixating eye was $19.0 \pm 2.1 \mathrm{PD}$ and of the

Table 1. Eye position in both the awakened state and the surgical plane of anesthesia

\begin{tabular}{lccc}
\hline & $\begin{array}{c}\text { Eye position } \\
\text { in awakened state }\end{array}$ & $\begin{array}{c}\text { Eye position } \\
\text { under anesthesia }\end{array}$ & $\begin{array}{c}\text { Amount of } \\
\text { divergence }\end{array}$ \\
\hline Orthophoria group $(\mathrm{N}=30)$ & $3.9 \pm 3.5 \mathrm{PD}$ & $31.4 \pm 7.6 \mathrm{PD}$ & $27.4 \pm 8.1 \mathrm{PD}$ \\
Exophoria group $(\mathrm{N}=30)$ & $5.6 \pm 3.5 \mathrm{PD}$ & $34.0 \pm 4.6 \mathrm{PD}$ & $36.6 \pm 11.7 \mathrm{PD}$ \\
Exotropia I group $(\mathrm{N}=15)$ & $24.9 \pm 4.7 \mathrm{PD}$ & $36.0 \pm 9.1 \mathrm{PD}$ & $11.1 \pm 10.2 \mathrm{PD}$ \\
Exotropia II group $(\mathrm{N}=15)$ & $47.1 \pm 6.5 \mathrm{PD}$ & $36.6 \pm 5.2 \mathrm{PD}$ & $-11.0 \pm 6.5 \mathrm{PD}$ \\
Esotropia group ( $\mathrm{N}=15)$ & $-17.9 \pm 6.4 \mathrm{PD}$ & $21.8 \pm 7.5 \mathrm{PD}$ & $39.7 \pm 8.0 \mathrm{PD}$ \\
& $\mathrm{P}=.000^{*}$ & $\mathrm{P}=.006^{*}, \mathrm{P}=.137^{+}$ & \\
\hline
\end{tabular}

(+: Divergence, -: Convergence)

${ }^{*}$ Multiple comparison between 5 groups, ${ }^{\dagger}$ Multiple comparison between 4 groups with the esotropia group excluded.

(Statistics by Kruskal-Wallis one-way ANOVA).

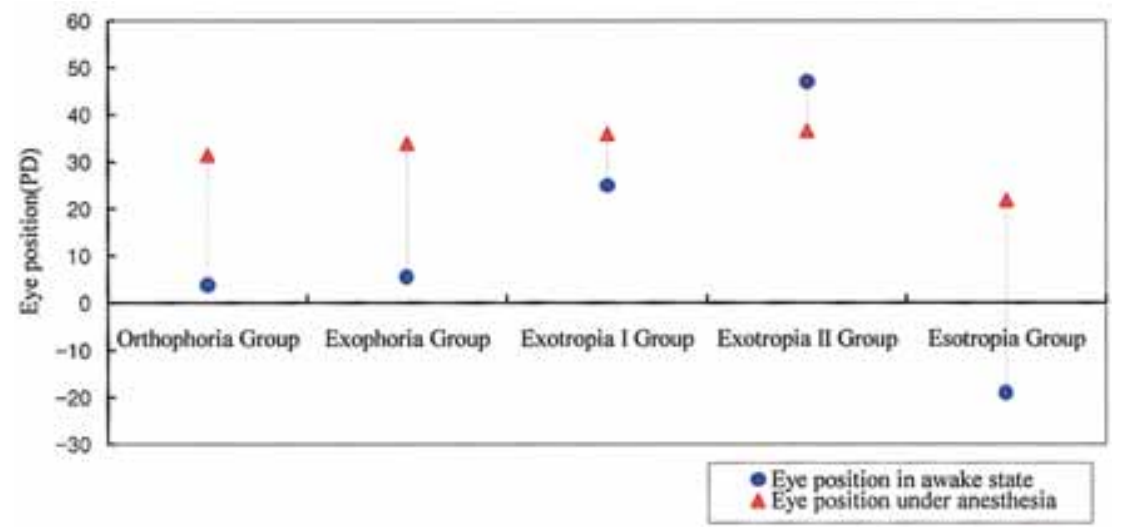

Fig. 2. Eye position in both the awakened state and the surgical plane of anesthesia. 


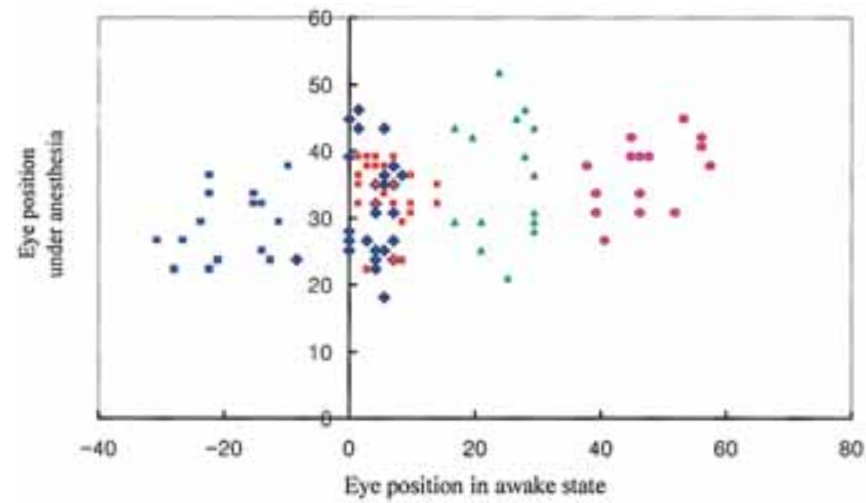

- Orthophoria Group

- Exophoria Group

- Exotropia I Group

- Exotropia II Group

- Esotropia Group

Table 2. Change of eye position of the right and left eyes in the orthophoria group $(\mathrm{N}=30)$

\begin{tabular}{lccc}
\hline & Right eye & Left eye & P-value \\
\hline Eye position in awakened state & $0.2 \pm 0.1 \mathrm{PD}$ & $0.3 \pm 0.1 \mathrm{PD}$ & $0.34^{*}$ \\
Eye position under anesthesia & $13.6 \pm 6.6 \mathrm{PD}$ & $13.6 \pm 5.0 \mathrm{PD}$ & $0.45^{*}$ \\
Amount of divergence & $13.4 \pm 6.5 \mathrm{PD}$ & $13.3 \pm 4.9 \mathrm{PD}$ & \\
\hline
\end{tabular}

(+: Divergence, -: Convergence)

*Statistics by t-test.

Table 3. Change of eye position of the fixating and nonfixating eyes in the exotropia group ( $N=30)$

\begin{tabular}{lccc}
\hline & Fixating eye & Nonfixating eye & P-value \\
\hline Eye position in awakened state & $1.7 \pm 3.6$ PD & $34.3 \pm 12.9$ PD & $<0.01^{*}$ \\
Eye position under anesthesia & $19.0 \pm 2.1$ PD & $18.2 \pm 6.4$ PD & $0.68^{*}$ \\
Amount of divergence & $17.3 \pm 5.7$ PD & $-16.1 \pm 6.5$ PD & \\
\hline
\end{tabular}

(+: Divergence, -: Convergence)

*Statistics by t-test.

Table 4. Change of eye position of the fixating and nonfixating eyes in the esotropia group ( $N=15)$

\begin{tabular}{lccc}
\hline & Fixating eye & Nonfixating eye & P-value \\
\hline Eye position in awakened state & $-1.6 \pm 4.7$ PD & $-17.7 \pm 0.9$ PD & $<0.01^{*}$ \\
Eye position under anesthesia & $14.4 \pm 4.8$ PD & $14.1 \pm 4.8$ PD & $0.71^{*}$ \\
Amount of divergence & $20.0 \pm 4.8$ PD & $31.8 \pm 8.9$ PD & \\
\hline
\end{tabular}

(+: Divergence, -: Convergence)

*Statistics by Mann-Whitney test.

nonfixating eye was $18.2 \pm 6.4 \mathrm{PD}$, with the difference between the two eyes not being statistically significant $(\mathrm{P}=$ 0.68) (Table 3).

In the esotropia group, before anesthesia, the fixating eye was $-1.6 \pm 4.7 \mathrm{PD}$ and the nonfixating eye was $-17.7 \pm 0.9$ $\mathrm{PD}$, and similar to the result for exotropia, the difference between the two eyes was statistically significant $(\mathrm{P}<0.01)$. After anesthesia, the divergence of the fixating and

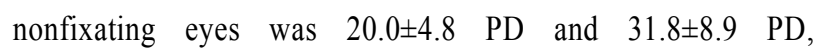

respectively. In the divergent position, the fixating eye was 14.4 \pm 4.8 PD and the nonfixating eye was $14.1 \pm 4.8 \mathrm{PD}$, with the difference between the two eyes not being statistically significant $(\mathrm{P}=0.71)$ (Table 4).

\section{DISCUSSION}

It is known that even the eye position that showed orthophoric position during the awakened state changed to 
various positions after general anesthesia. In 1890, Grut ${ }^{1}$ reported that during sleep, death, blindness, and after anesthesia, the eye position was divergent, and thus the anatomical position of the eye at the resting state was divergent. Brenin $^{8}$ reported that the eye position during anesthesia is divergent, and $\mathrm{Cattel}^{9}$ that the eye position after death is diverse and not always divergent. Moses ${ }^{10}$ reported that after anesthesia, the eye was centrally positioned, and Moller $^{2}$ reported that the squint angle was reduced in esotropia patients following the administration of morphine or atropine during general anesthesia. Recently, in Korea, it has been reported that the eye position after anesthesia generally showed a divergent tendency. ${ }^{3-7}$ Regarding the cause of this divergence of eye position after general anesthesia, Burford $^{11}$ reported that after general anesthesia, the tension of the extraocular muscle was lost and the action potential did not appear. Brenin ${ }^{8}$ reported that after general anesthesia, the slowdown and blocking of the neurotransmission to the extraocular muscles occurred and thus the eye was in the anatomically resting position, and at that time, due to the anatomical morphology of the orbital structure, the eyes became divergent. Worth and Chavasse ${ }^{12}$ reported that this was due to the residual divergence of the orbital axis that occurred during anesthesia.

In regard to the eye position after general anesthesia, Apt and Isenberg ${ }^{19}$ reported that the divergence was 11.9 PD in exotropia and 43.1 PD in esotropia in 317 strabismus patients, and Romano and Gabriel ${ }^{20}$ that 66 patients showed divergence, and 9 showed no change in 77 strabismus patients. Lee et $\mathrm{al}^{3}$ reported that all 42 study subjects showed divergence, with a divergence of $13.1 \mathrm{PD}$ in exotropic patients and 22.7 PD in esotropic patients. In exotropia patients, as the strabismus angle before anesthesia was smaller, and in esotropia patients, as the squint angle before anesthesia was larger, the divergence of the eye was larger during general anesthesia. Ohmi et $\mathrm{al}^{21}$ reported that in 62 exotropia patients, 60 showed divergence and 2 showed no change, and that in 80 exotropia patients, 57 showed divergence, 17 showed convergence, and 6 showed no change. Mitsui et $\mathrm{al}^{22}$ reported that among 10 esotropia patients, 9 showed divergence and 1 showed convergence. In Korea, Lim et $\mathrm{al}^{6}$ reported that all 27 esotropia patients showed divergence, and that in 140 exotropia patients, 6 showed divergence, 107 showed convergence 27 showed no change. In our study, orthophoria, exotropia, and esotropia showed divergence during general anesthesia; however, among exotropia patients, the group over 30 PD showed convergence. The cause is that in this esotropia II group, as the fixating eyes showed divergence and the nonfixating eyes showed convergence, the sum of the fixating and nonfixating eyes showed convergence. In addition, measuring the divergence of the right and left eyes in orthophoria patients separately, the eye positions were not different $(\mathrm{P}=$ 0.45). In the exotropia and esotropia groups, the differences between fixating and nonfixating eyes were not detected, which suggests that regardless of the presence of strabismus, the resting state of the fixating and nonfixating eyes is defined, and that it is not different between the two eyes unless the orbital bony structure is asymmetric. Hence, as described by Duke-Elder, ${ }^{24}$ the eye after anesthesia is switched to the absolute resting state of the eye that does not reflect the type and degree of strabismus as well as the dominant eye in the awakened state, and they converged at the position of 25 35 PD. Hence, since the degree of deviation of the orbit during the awakened state is not reflected in the eye position after general anesthesia, it appears that the eye position after general anesthesia is not a factor to be considered for the determination of the eye to be operated on, the surgical method, or the surgical degree.

On the other hand, in esotropia, the divergence rate after general anesthesia was larger than that of the other groups. However, the eye position after anesthesia was more to the medial side than in the other groups because the neurotransmission of the medial rectus muscle is bigger than that of the lateral rectus muscle, the tension relaxation of the extraocular muscles is not sufficient, and neurotransmission of the medial rectus muscle remains. Therefore, due to the excess neurotransmission of the medial rectus muscle for a long period, the ratio of the medial rectus muscle fiber against the lateral rectus muscle becomes unbalanced, and thus the tension of the lateral rectus muscle is relaxed sufficiently while a portion of the medial rectus muscle is not relaxed, which may cause the insufficient divergence.

Different from the eye position measurement during the awakened state, the eye position after general anesthesia was measured by Hirshberg or Krimsky prism test method in the studies reported so far. ${ }^{5,6,19,20}$ However, in our study, the squint angle was measured by applying the pictures of the eye position taken by digital camera both during the awakened state and after general anesthesia. The reasons 
were that first, the error due to the difference in measuring method could be reduced by standardizing the method for eye position measurement both during the awakened state and after general anesthesia, second, in the measurement of the squint angle after general anesthesia by applying Krimsky prism tests, the location of the corneal reflex on the center of the cornea was confirmed by the estimation of the investigators and thus the measurement error may have been substantial, third, the separate measurement of the eye position of the right and left eyes was feasible because the change of the eye position of the right and left, fixating and nonfixating eyes could be evaluated independently, and fourth, the angle kappa could be measured. Hence, for distinguishing between strabismus and phoria, although using the Hirshberg method and digital photography for the measurement is less accurate than the alternative prism cover test, it is advantageous in terms of providing a uniform measurement method for the eye position.

In our study with the eye position measurement based on the corneal reflex, the distance from the nasal limbus to the corneal reflex was measured. The eye position measurement by corneal reflex consists of two methods: one based on the corneal nasal limbus and the other based on the center of the pupil. Barry and Backes ${ }^{25}$ reported that the former method is more accurate than the latter method because it is difficult to determine the accurate center of the pupil with the pupils skewed on pictures, and because the measurement based on the center of the pupil requires the conversion of other values. In our study, the center of the pupil was difficult to determine in many cases, and in the eyes with severe deviation the distance from the center of the pupil to the corneal reflex measured photographically was shorter than the actual distance, producing a larger error. Therefore, the distance based on the nasal limbus of the cornea was measured. The length actually measured was converted by the Hirschberg ratio, $14 \mathrm{PD} / \mathrm{mm}$, which is based on the report by Hirschberg in $1886^{18}$ indicating that $1 \mathrm{~mm}$ represents $8^{\circ}$ deviation. Subsequently, several investigators have reported slightly different values, and recently Brodie ${ }^{27}$ reported the deviation of $21 \mathrm{PD}$ per $\mathrm{mm}$. In Korea, Lee and $\mathrm{Lee}^{26}$ reported the deviation of $15.2 \mathrm{PD}$ per $\mathrm{mm}$.

In all orthophoria patients under the awakened state except one, the corneal reflex was in the medial side of the center of the cornea. The case with the corneal reflex on the lateral side was confirmed to be negative angle kappa. In the orthophoria group, the average distance from the center of the cornea to the corneal reflex was positive, $3.9 \pm 3.5 \mathrm{PD}$, and 1 out of 30 patients showed a negative angle kappa of -11.2 PD. Generally according to Donders, ${ }^{23}$ the angle kappa shows a positive value of $5.082^{\circ}$ on average. On the other hand, in our study, the angle kappa was not adjusted during the measurement of the squint angle, except for orthophoria. For the adjustment of the angle kappa in the strabismus patient group, the angle kappa of each eye was measured after covering one eye, from which the value from the squint angle obtained during the staring with both eyes was subtracted. However, as the angle kappa of the orthophoria group was not substantial, at $3.9 \pm 3.5 \mathrm{PD}$, it is believed that it did not affect the overall result significantly.

During the induction phase of anesthesia, the depolarizing muscle relaxant succinylcholine and the non-depolarizing muscle relaxant vecuronium were used. In regard to succinylcholine action, France et $\mathrm{al}^{13}$ reported that succinylcholine induced the unpredictable contraction of the extraocular muscle for at least 15 minutes after intravenous injection. Mindel et $\mathrm{al}^{14}$ reported that before and during anesthesia, the administration of succinylcholine selectively induced the contraction of the muscle fiber with complex neuromuscular junction in the outer muscle layer, that stimulation was blocked in the muscle fiber with a single neuromuscular junction in the inner layer of muscle, and thus that the eye position becomes the position of the conscious state. Lingua et $\mathrm{al}^{15}$ reported that during anesthesia, the eye position treated with succinylcholine immediately after surgery correlated to the eye position one week after surgery. Similarly, in Korea, Lee and $\operatorname{Jin}^{16}$ reported that during general anesthesia, the eye position after the presurgical injection of succinylcholine was correlated with that one day before surgery. Although the effect of succinylcholine on the eye position is substantial in such manners, Lingua et $\mathrm{al}^{17}$ reported that the reactive time of succinylcholine is short in that it is degraded and inactivated fast within 1 3 minutes. In our study, the squint angle was measured 20 minutes after anesthesia when the effect of succinylcholine had worn off. To prevent the ion passage becoming unopenable and the depolarization due to the competitive binding of acetylcholine to the acetylcholine receptor, the representative, non-polarizing, muscle relaxant, vecuronium $^{28}$ that blocks the neuromuscular transmission pathway was administered to induce sufficient muscle 
relaxation.

In conclusion, regardless of the eye position during the awakened state, the eye position after general anesthesia is divergent or convergent, resulting in a pattern of convergent to divergent position of 25 35 PD. Therefore, before anesthesia the eye position was unpredictable, and it was impossible to distinguish between the fixating and nonfixating eyes in manifest strabismus. This may be because the eye position under general anesthesia is not related to that during the awakened state. Additional studies are required to investigate the correlation of eye position after strabismus surgery to that in the awakened state from anesthesia.

\section{REFERENCES}

1. Grut HE. A contribution to the pathogeny of concomitant squinting. Trans Ophthalmol Soc UK 1890;10:1.

2. Möller PM. Influence of anesthesia and premedication on the squint angle. Acta Ophthalmol 1958;36:499-501.

3. Lee SY, Seong GJ, Kim HB. Eye position of strabismus patients under general anesthesia. J Korean Ophthalmol Soc 1987;28:117-20.

4. Kim SJ, Oh JS, Kim JM. A study of the change in angle deviation under general anesthesia in horizontal strabismus patients. J Korean Ophthalmol Soc 1989;30:115-22.

5. An GJ, Cho YA, Jung HR. The change of the squint angle under general anesthesia using pancuronium bromide (Myoblock ${ }^{\circledR}$ ). J Korean Ophthalmol Soc 1989;30:773-8.

6. Lim ST, Kim SJ, Park YG. A clinical study: change of the eye position under general anesthesia. J Korean Ophthalmol Soc 1995;36;2243-51.

7. Lee DS, Kim SY. Eye position of orthophoric patients under general anesthesia. J Korean Ophthalmol Soc 2001;42:1303-8.

8. Brenin GM. The position of rest during anesthesia and sleep. Arch Ophthalmol 1957;57:323-6.

9. Cattel HW. Post-mortem pathology. Philadelphia: JB Lippincott, $1905 ; 73$.

10. Moses RA. Adler's physiology of the eye. St. Louis: CV Mosby, $1970 ; 75$.
11. Burford G. Involuntary eyeball motions during anesthesia and sleep, relationship to cortical rhythmic potentials. Anesth Anal 1941;20:191.

12. Worth C, Chavasse B. Squint, 9th ed. London: Bailliere Tyndal \& Cox, 1959;714-8.

13. France NK, France TD, Woodburn JD Jr, Burbank OP. Succinylcholine alteration of the forced duction test. Ophthalmology 1980;87:1282-7.

14. Mindel JS, Raab EL, Eisenkraft JB, Teutsch G. Succinyldicholineinduced return of the eyes to the basic deviation. Ophthalmology 1980;87:1288-95.

15. Lingua RW, Azen S, Szirth B, et al. Succinylcholine as a predictor in strabismus surgery. J Pediatr Ophthalmol Strabismus 1983;20:145-8.

16. Lee IS, Jin YH. Succinylcholine induced - ocular position under the general anesthesia. J Korean Ophthalmol Soc 1987;28:387-91.

17. Lingua RW, Azen SP, Walonker F, et al. A comparison of the succinylcholine induced ocular position and the postoperative alignment in strabismus. J Pediatr Ophthalmol Strabismus 1986; 23:69-73.

18. Hirschberg J. Beitrage zur Lehre vom Schielen und von der Schieloperation. Centralbl prakt Augenh 1886;10:5-9.

19. Apt L, Isenberg S. Eye position of strabismus patients under general anesthesia. Am J Ophthalmol 1977;84:574-9.

20. Romano P, Gabriel L. Intraoperative adjustment of eye muscle surgery. Correction based on eye position during general anesthesia. Arch Ophthalmol 1985;103:351-3.

21 Ohmi E, Oguri K, Yoshiya I, Kitamura S. Eye position of squinting eyes during general anesthesia. Acta Ophthalmol Jap 1975;79:540.

22. Mitsui Y, Tamura O, Hirai KI, et al. An electromyographic study of esotropia. Br J Ophthalmol 1981;65:161-6.

23. Donders FC, Moore WD. On the anomalies of accommodation and refraction of the eye. London: New Sydenhan Society, 1864.

24. Duke-Elder S. System of ophthalmology. Vol. 6. St. Louis: CV Mosby, $1973 ; 96$.

25. Barry JC, Backes A. Limbus versus pupil center for ocular alignment measurement with corneal reflexes. Invest Ophthalmol Vis Sci 1997;38:2597-607.

26. Lee JA, Lee HY. New method for measurement of strabismic angle using corneal reflex and photograph. J Korean Ophthalmol Soc 2002;43:1988-92.

27. Brodie SE. Photographic calibration of the Hirschberg test. Invest Ophthalmol Vis Sci 1987;28:736-42.

28. McKenzie AG. Prelude to pancuronium and vecuronium. Anaesthesia 2000;55:551-6 